\title{
Winds and Shocks in Galaxy Clusters: Shock Acceleration on an Intergalactic Scale
}

\author{
T. W. Jones*, Francesco Miniati*, \\ Dongsu Ryu, ${ }^{\dagger}$ and Hyesung Kang \\ * Department of Astronomy, University of Minnesota, Minneapolis, MN 55455, USA \\ ${ }^{\dagger}$ Dept. of Astronomy and Space Science, Chungnam National Univ., Daejeon 305-764, Korea \\ "Department of Earth Science, Pusan National University, Pusan 609-735, Korea
}

\begin{abstract}
We review the possible roles of large scale shocks as particle accelerators in clusters of galaxies. Recent observational and theoretical work has suggested that high energy charged particles may constitute a substantial pressure component in clusters. If true that would alter the expected dynamical evolution of clusters and increase the dynamical masses consistent with hydrostatic equilibrium. Moderately strong shocks are probably common in clusters, through the actions of several agents. The most obvious of these agents include winds from galaxies undergoing intense episodes of starbursts, active galaxies and cosmic inflows, such as accretion and cluster mergers. We describe our own work derived from simulations of large scale structure formation, in which we have, for the first time, explicitly included passive components of high energy particles. We find, indeed that shocks associated with these large scale flows can lead to nonthermal particle pressures big enough to influence cluster dynamics. These same simulations allow us also to compute nonthermal emissions from the clusters. Here we present resulting predictions of $\gamma$-ray fluxes.
\end{abstract}

\section{INTRODUCTION}

Clusters of galaxies are very important probes for cosmology, since they are the largest bound systems in the universe. They represent the nonlinear development of large-scale perturbations in the early Hubble flow. Matter, energy and entropy can be added from outside through infall or cluster mergers, but with the exception of a small fraction of the energy as photons, neutrinos and ultra high energy cosmic-rays, nothing leaves clusters once they form. Thus, they provide unique records of the history of the universe. The statistics of cluster masses and their dynamical properties, including, for instance, the relative proportions of baryonic and nonbaryonic matter, are commonly used to test basic cosmological models [1]. While galaxies are the most obvious constituents of clusters in visible light, most 
of the matter in clusters is nonbaryonic, and even the baryonic matter is primarily contained within the diffuse intracluster medium (ICM), rather than the galaxies. The temperature and density distribution of the ICM gas directly reflects the dynamical state of the clusters, as well as holding a history of important dissipative processes encountered by the gas [39]. Elemental abundances in the ICM are seen as key indicators of the star formation histories and of galaxy evolution, more generally $[43,53]$. The dynamical states of clusters have also received much attention. While cluster ICMs sometimes appear relaxed, often the situation is quite different, with clear indications of high speed flows $[13,15,32,33,40]$ demonstrating that cluster environments can be violent.

The possible importance of nonthermal components in the ICM has recently raised great interest. There is growing evidence that magnetic fields [9,35], and high energy charged particles $[6,27,38]$ may constitute significant dynamical components of the ICM in at least some clusters. If these components are generally strong, they would impact on a wide range of issues, beginning with estimates of cluster masses derived from assumptions of ICM hydrostatic equilibrium. In addition, since high energy charged particles and magnetic fields do not readily radiate away their pressures, they could tend to inhibit cooling flows. Radio, EUV and hard $\mathrm{X}$-ray emissions resulting from energetic electrons have already stimulated much discussion about cluster physics as well as the evolution of the clusters and their galaxies $[4,19,34,45,49,53]$. While high energy $\gamma$-rays have not yet been detected from clusters, recent estimates of $\gamma$-ray luminosities from high energy particle interactions in the nearest rich clusters, such as Coma, are within the range of what may be detected in the next generation of $\gamma$-ray observatories $[6,10]$.

Our focus here is the possible relationships between shock waves generated by high speed flows in clusters and the origins of the high energy charged particles. For convenience, we call all such particles cosmic-rays or "CRs". A few basic considerations of the CRs are necessary to set the stage. So far direct observations reveal only the presence of $\mathrm{CR}$ electrons, although one generally assumes that protons are present with at least comparable numbers and greater energy content [38]. Relativistic protons below energies where photo-pion production through interaction with the cosmic microwave background (CMB) becomes important, $\left(\sim 10^{9.5} \mathrm{GeV}\right)$ do not lose significant fractions of their energy in a cluster over a Hubble time [4]. Also, up to somewhat lower energies they should scatter off magnetic irregularities sufficiently to remain trapped in the cluster for a similar duration [53]. Relativistic electrons, on the other hand can only survive Coulomb losses and radiative losses for more than $\sim 1$ Gyr in a relatively narrow energy band centered roughly around $50 \mathrm{MeV}$ [50]. Thus, observed relativistic electrons must be freshly accelerated, reaccelerated or be introduced as decay products of other interactions $[8,12,16]$.

The Coma cluster provides a convenient scale for the energetics involved in producing and containing the CRs. The dynamical mass estimates of Coma are a bit over $10^{15} M_{\odot}$ [18], leading to an ICM mass $\sim 10^{14} M_{\odot}$. With a mean ICM temperature of $8 \mathrm{keV}$ [55] the total thermal energy in the ICM $\sim 4 \times 10^{63} \mathrm{erg}$. Observed 
excess EUV emission from Coma, interpreted as inverse Compton scattered CMB photons, leads to an estimated $\gtrsim 10^{61} \mathrm{erg}$ in total CR electron energy and, through conventional arguments, $\gtrsim 10^{63} \mathrm{erg}$ in protons $[38,49]$. If we accept that figure for argument and assume the CR protons were supplied continuously over a Hubble time the mean CR energy input rate would be $\sim 3 \times 10^{45} \mathrm{erg} / \mathrm{sec}$. By comparison the energy input to CRs in our own galaxy is only about $10^{41} \mathrm{erg} / \mathrm{sec}[14,17]$. This estimate of CR energy in Coma makes it clearly very important dynamically, and the implied rate of energy input severely restricts the range of possible accelerators.

\section{LARGE-SCALE SHOCKS AS ACCELERATORS}

There are likely to be many particle accelerators in clusters, but only a few seem really capable of accounting for the CR energy content implied above for clusters like Coma. Thus, for example, while second order Fermi acceleration from MHD turbulence in the ICM might conceivably play a role in electron acceleration $[8,16]$, that would not seem to be a very fruitful approach to accounting for proton CRs, if their energy content exceeds $\sim 10^{63} \mathrm{erg}$ in rich clusters. In fact, most current models for proton acceleration depend on first order Fermi acceleration resulting from CR diffusion near shocks. This so-called "diffusive shock acceleration", or DSA, can be efficient enough to put into CRs several tens of percent of the total energy flux through a shock [3]. Following that lead, the initial task becomes one of identifying shocks that dissipate enough energy to explain the energetics suggested for Coma and by implication other rich clusters. It turns out that large scale shocks are probably fairly common in ICMs, so it may indeed be reasonable to expect a substantial energy in CR protons there. Electrons are more difficult to accelerate and maintain than protons. First, they radiate energy much more rapidly [50]. In addition, they are harder to get started in most accelerators, because of their small gyroradii at suprathermal energies. Still, from galactic CRs and other environments, such as radio galaxies, we have abundant evidence that electrons can be accelerated. Also, inelastic proton-proton interactions with the ICM can generate a significant secondary electron-positron component through pion decay $[11,12]$.

Several candidate classes of large shock structures come to mind immediately in clusters. For example, the facts that ICM gas is generally enriched with nucleosynthetic products to $10-30 \%$ of the solar value [43] and that starburst galaxies like M82 produce strong winds, have been cited to support the idea that termination shocks in galactic winds may have been common enough during early "bright phases" of galaxy evolution to accelerate a substantial high energy CR population $[26,53]$. Starburst-like energy deposition rates are probably necessary to generate winds inside rich clusters, in order to overcome the substantial thermal pressure of the ICM [53]. If one takes an ICM Fe mass $\sim$ few $\times 10^{11} M_{\odot}[7]$ and assumes it resulted from supernovae, then $\sim 10^{12}$ events, corresponding to about $10^{63}$ erg of 
kinetic energy would have been released into a rich cluster like Coma [54]. That is close to the CR energy content required, but does not yet account for a realistic conversion efficiency. Even if we assume $\sim 100 \%$ of the supernova energy ends up as kinetic energy in the winds and that we can carry over from galactic supernova
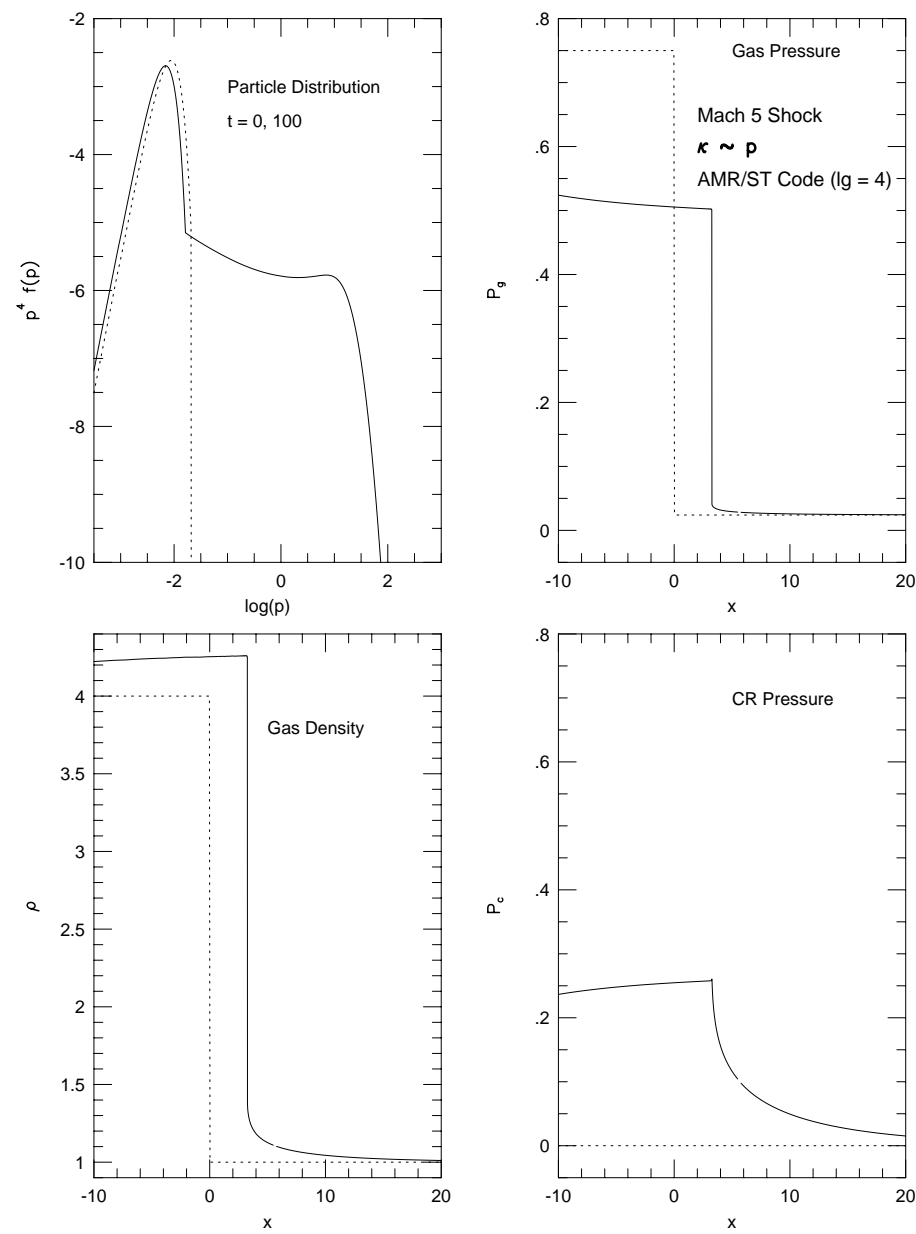

FIGURE 1. Properties of a simulated Mach 5 shock including backreaction from nonthermal particles accelerated by DSA after being injected through "thermal leakage". The momentum distribution of the particles just downstream of the shock is also shown. The dotted lines show initial conditions, while the solid/dashed lines show properties after CR protons have been accelerated to energies $\sim 100 \mathrm{GeV}$ and the shock structure is almost steady. Bohm-like spatial diffusion, $\kappa \propto p$, is assumed. 
a CR acceleration efficiency $\sim 10-20 \%$ [22], this model seems to struggle to account for the very large energy contents if the recent EUV observations represent inverse Compton emission and the implied CRs fill the entire cluster [38]. On the other hand, these winds may still be effective accelerators or reaccelerators at a somewhat lower level, as well as contributors to the ICM magnetic field [34].

CRs escaping from radio galaxies have long been seen as potential sources of relativistic particle populations in clusters and sometimes beyond [19,23]. These CRs are usually seen to be accelerated by the terminal shocks of the jets powering the radio lobes, but it also seems plausible that the bow shocks of at least the most powerful radio jets in cluster environments might be effective accelerators. Energetically, radio galaxies come close to required input levels if we can assume on average that a rich cluster contains at least one at all times [4]. The most luminous radio galaxies are estimated to be driven by jet kinetic powers $\gtrsim 10^{45} \mathrm{erg} / \mathrm{sec}$ [2], which is comparable to our earlier estimated average rate of CR production for Coma. Individual radio galaxy lifetimes are generally estimated at $\sim 10^{8}$ years $[2]$, so such a cluster would need as many as $\sim 10^{2}$ luminous galaxies capable of generating a powerful radio jet or a fairly high duty cycle in a smaller number of galaxies. On the other hand, this localized source model may have some difficulty explaining the diffuse radio halos seen in some luminous clusters [37], including Coma, because of large energy loss rates for high energy electrons and because low energy electrons probably cannot diffuse very far from their source [23]. Reacceleration can, of course, reduce or eliminate this issue [8]. Similarly, if the energy were deposited in high energy protons the difficulty is reduced. Then the nonthermal emissions observed so far would presumably be explained through secondary electrons. There are also suggestions that the relatively light plasma in radio lobes may remain confined in buoyant "ghost" bubbles, so that they can be reenergized and illuminated at later times by passage of other large scale shocks [20].

Another scenario gaining some strong recent support is CR acceleration at very large and long-lived shocks resulting from cosmic structure formation. The likely existence of strong accretion shocks several megaparsecs from cluster cores has been recognized for a long time $[5,47]$, and they have been suggested as possible sources of ultra-high energy CRs $[29,30]$ as well as seeds for the ICM magnetic field [36]. Shocks resulting from discrete cluster merger events have also been recognized through X-ray structure in clusters [13,40], and cited as particle accelerators to account for diffuse cluster radio halos $[21,52]$ or so-called "relic" radio sources $[45,44]$. The overall energetics of these shocks is generally acceptable for the production of the CRs needed. Typical flow speeds in and around clusters will be $v_{f} \sim\left(2 G M_{c l} / R_{c l}\right)^{1 / 2} \sim 2 \times 10^{3} \mathrm{~km} / \mathrm{sec}$, leading to an available power $\sim \rho_{c l} v_{f}^{3} R_{c l}^{2} \sim 10^{46} \mathrm{erg} / \mathrm{sec}$, using $M_{c l} \sim 10^{15} M_{\odot}$ and $R_{c l} \sim 2 \mathrm{Mpc}$. Accretion shocks far from cluster cores can be of very high Mach number and are responsible for initial heating of the ICM. They appear, however, to be less important as potential sources of CRs inside clusters than weaker, "internal" shocks associated with mergers and other flows penetrating deeper into the clusters. The reason is that the latter shocks repeatedly process the ICM material, whereas the accretion 
shocks do it only once.

Miniati et al [41] explored the statistics of shock heating in both SCDM and $\Lambda \mathrm{CDM}$ cosmologies. They found that far more mass gets processed through the internal shocks than the accretion shocks. Their analysis showed that the most common shock encounters by the ICM material involve shock Mach numbers less than 10, with the peak around $M \sim 5$. That is significant, since such shocks are strong enough to transfer as much as $20-30 \%$ of the postshock pressure to CRs. On the other hand such shocks are only modestly modified in their structures through backreaction of CRs diffusing upstream. Thus, so-called "test particle" estimates of the $\mathrm{CR}$ distributions are a reasonable first guess to what we may expect. Figure 1 illustrates the evolution of a CR modified Mach 5 shock as computed from a fully nonlinear DSA simulation carried out using methods detailed elsewhere [31].

\section{SIMULATING CR ACCELERATION IN COSMIC STRUCTURE FORMATION}

So far, only a few clusters have shown direct evidence of large shocks. However, recent cosmic structure formation simulations have demonstrated that large scale shocks in clusters are probably much more common and much more complex than these simple perceptions suggest [41]. Since clusters tend to form at the intersections of cosmic filaments, they accrete matter in very unsteady and nonisotropic patterns. In addition to discrete cluster mergers, general, larger scale flows and shocks associated with them propagate down the filaments and through the clusters. When cluster mergers take place the accretion shocks associated with the

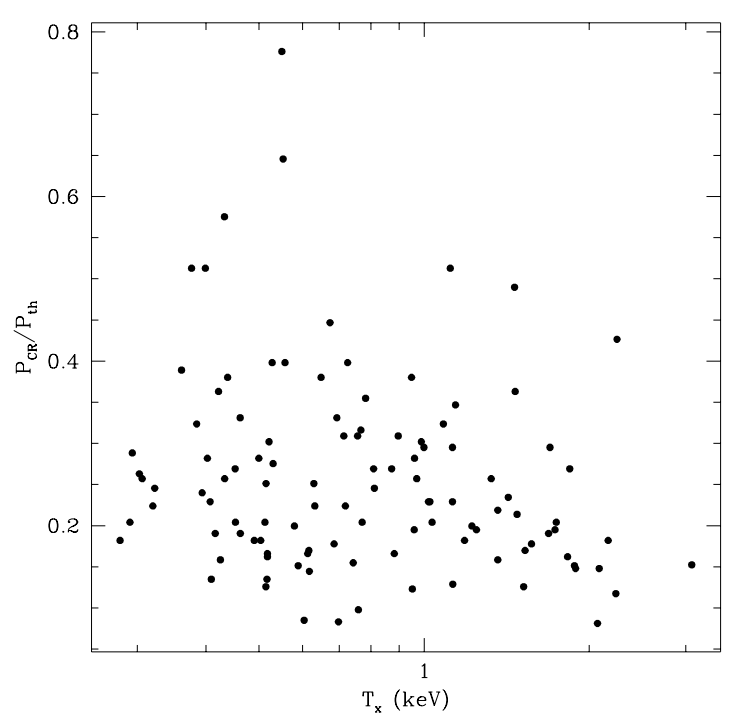

FIGURE 2. Mean $P_{C R} / P_{\text {th }}$ measured in simulated clusters inside $r=0.5 \mathrm{Mpc} / \mathrm{h}$ at $z=0$. 
individual clusters add to the shocks that form in direct response to the merger. The net result of all of this is a rich web of shocks, which often penetrates into the inner regions of the clusters.

The recent detections of shocks from merger events has been noted above. But the existence of accretion shocks is not necessarily easy to detect directly. On the other hand, infalling clouds of unshocked, warm gas outside clusters may be identified through the absorption lines of quasars located inside clusters. This warm low density gas of $10^{4}-10^{5} \mathrm{~K}$ is photoionized by the diffuse radiation from the hot postshock gas and the diffuse cosmic background radiation. In some studies $[24,56]$, the C IV absorption systems of quasar emission lines with $\left|z_{a b s}-z_{Q S O}\right|<3000 \mathrm{~km} \mathrm{~s}^{-1}$ are interpreted as clouds associated with rich clusters where the quasars reside. The characteristics of these systems of C IV absorbers are different from those of the typical intervening $\mathrm{C}$ IV absorbers. It has been noted that the velocity difference is unexpectedly large compared to the typical velocity dispersion of galaxies $\left(400-1200 \mathrm{~km} \mathrm{~s}^{-1}\right)$ in rich clusters [24]. An accretion velocity, however, is a bit larger than the galaxy velocity dispersions, since it is given by $v_{a c c}=1.31 \times 10^{3} \mathrm{~km} \mathrm{~s}^{-1}\left(T_{c l} / 6.06 \mathrm{keV}\right)^{1 / 2}$, for example, in an Einstein-de Sitter universe $[5,46]$. Thus it is possible that these absorption systems are in fact the infalling clumps of gas upstream of the accretion shock.

Large shocks may be very efficient sources of CRs in clusters. We now outline a computational study of this phenomenon as revealed in a simulation by Miniati [42]. Briefly, this calculation used an Eulerian "TVD" hydro + N-body cosmology code [48] with passive treatments of magnetic fields [36] and CRs added. The CR protons and electron populations were accelerated according to DSA test-particle theory, then evolved to include adiabatic, radiative and Coulomb energy losses up to $10^{6} \mathrm{GeV}$, using methods similar to those described previously [25]. The upper
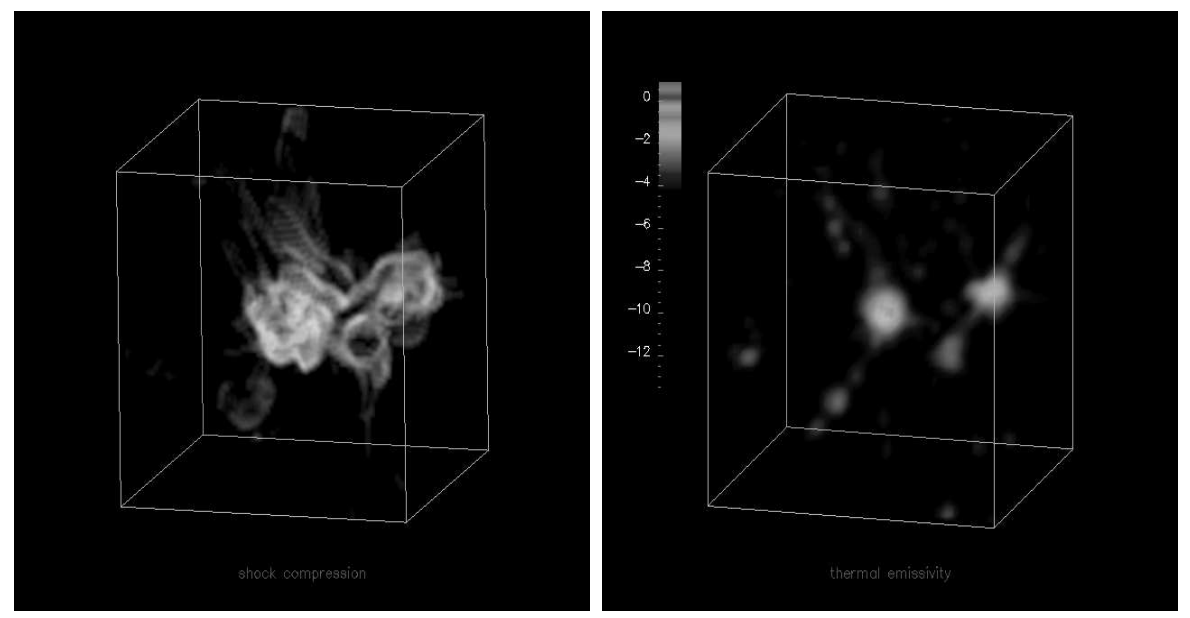

FIGURE 3. Volume renderings of clusters formed in a SCDM simulation. Left: Shock surfaces. Right: Thermal bremsstrahlung emissivity. A $14 \mathrm{Mpc} / \mathrm{h}^{3}$ volume is shown at $\mathrm{z}=0$ from a 50 $\mathrm{Mpc} / \mathrm{h}^{3}$ simulation. 
energy limit was chosen so spatial diffusion could be neglected in order to reduce computational costs, and since most observable emissions can be studied in this regime.

Secondary electron production due to pion decays, was also included. CRs were injected at shocks according to the "thermal leakage" model described by Kang \& Jones [28]. Roughly speaking, a small fraction of the downstream thermal particles, whose velocities are large enough for them to escape back across the shock, are assumed to be "injected" into the nonthermal particle population, whence they are subject to DSA. A $50 \mathrm{Mpc} / \mathrm{h}^{3}$ periodic section of a SCDM universe was simulated in this case on a $256^{3}$ grid, with $\mathrm{h}=0.5, \Omega_{M}=1, \Omega_{B}=0.13$ and $\sigma_{8}=0.6$.
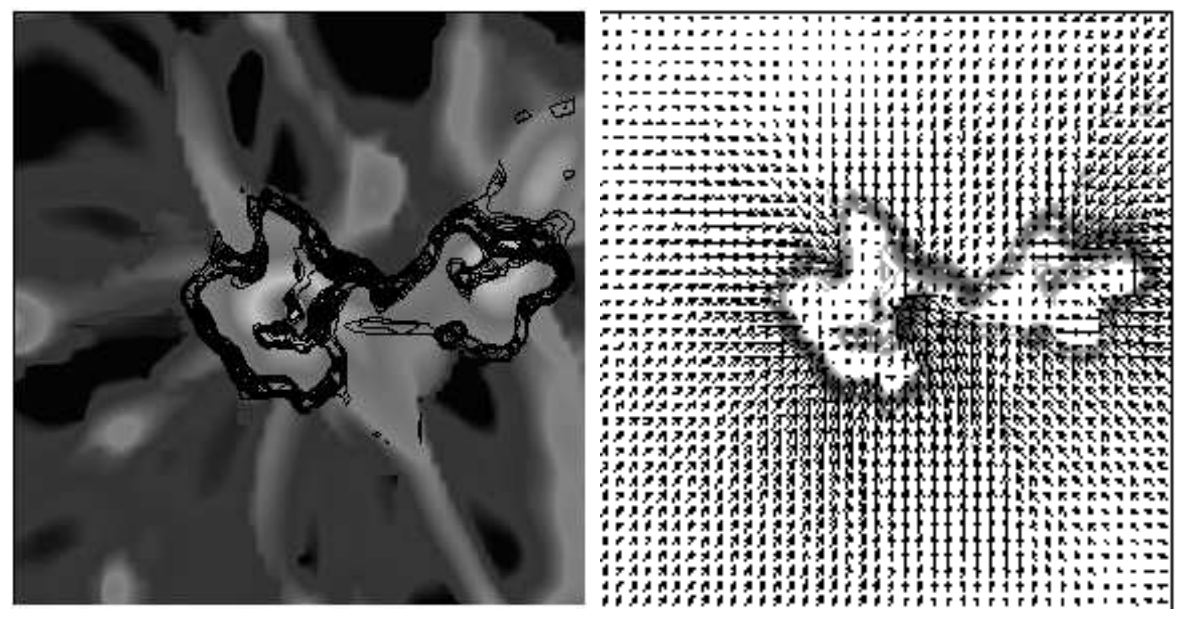

FIGURE 4. Vertical slices through the clusters shown in Figure 1. Left: Thermal bremsstrahlung emissivity with shock surfaces superposed. Right: Shock surfaces superposed on the projected flow velocity field.
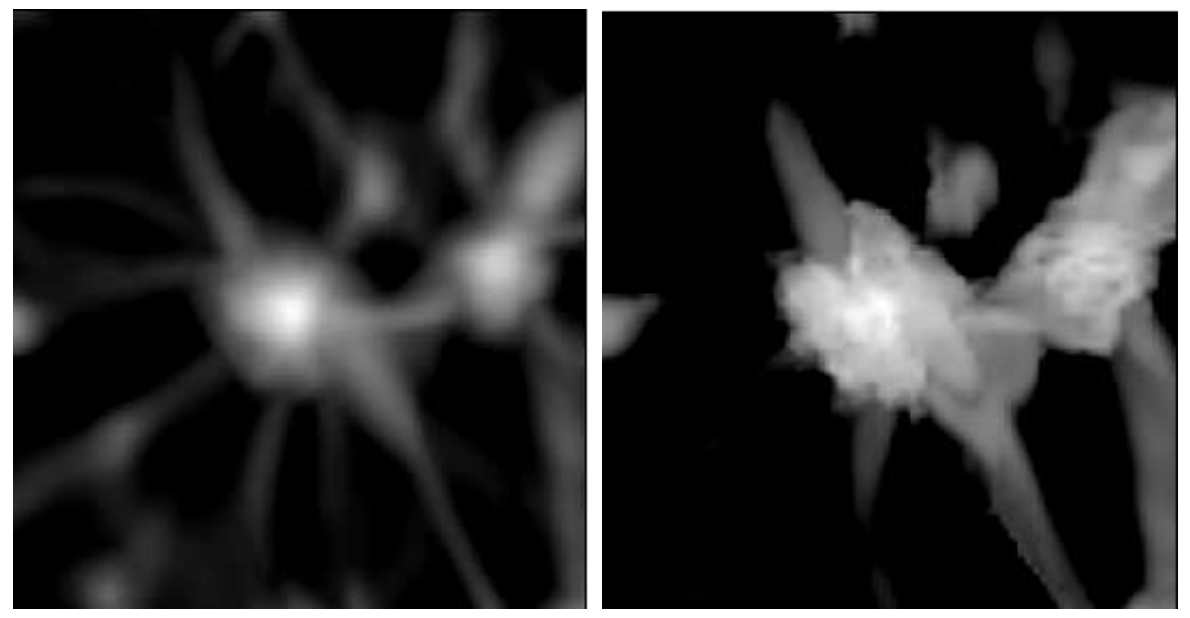

FIGURE 5. Vertical slices as in Figure 3. Shown are Left: Log thermal proton density. Right: Log CR proton density. 
Figure 2 shows the mean ratio of CR pressure to thermal pressure in the central regions of clusters in this simulation. These results are indicative only, since the CRs were not coupled back dynamically in cluster evolution. The mean ratio, $P_{C R} / P_{t h} \sim 0.25$, however, consistent with the results of nonlinear Mach $5 \mathrm{CR}$ shock simulations mentioned earlier. There is considerable scatter, since each cluster has a unique shock history. These nonthermal pressures are high enough that they would produce non-negligible influences on the internal cluster dynamics. Clearly, future simulations should consider those effects self-consistently.

The shock structures associated with the clusters are very complex. Figure 3 isolates from the simulation a $14 \mathrm{Mpc} / \mathrm{h}^{3}$ cube at $\mathrm{z}=0$, centered on a pair of interacting clusters. It shows clearly the web of shock surfaces in and around the clusters. The manner in which these shocks have formed and the considerable degree to which they penetrate into the cluster cores is shown in Figure 4. Here we have taken a vertical slice through the clusters in Figure 3, and show the intersections of the shock surfaces (contours of $\nabla \cdot v$ ) with the slice plane. These are superposed onto the thermal bremsstrahlung X-ray emissivity and projections of the vector velocity field. Associated cosmic filamentary features are evident, as well as the fact that strong flows are focussed along the filaments and directed at the clusters. Where those flows enter the clusters shocks sometimes penetrate deeply into the cores.

Figure 5 provides a comparison of gas and CR distributions in the same plane. On the left we show the spatial distribution of thermal protons, and on the right the distribution of $\mathrm{CR}$ protons. While there is a crude correspondence between the CRs and the gas, they clearly are not identical. The most obvious distinction is the existence of sharp edges in the CR distribution, reflecting their injection at

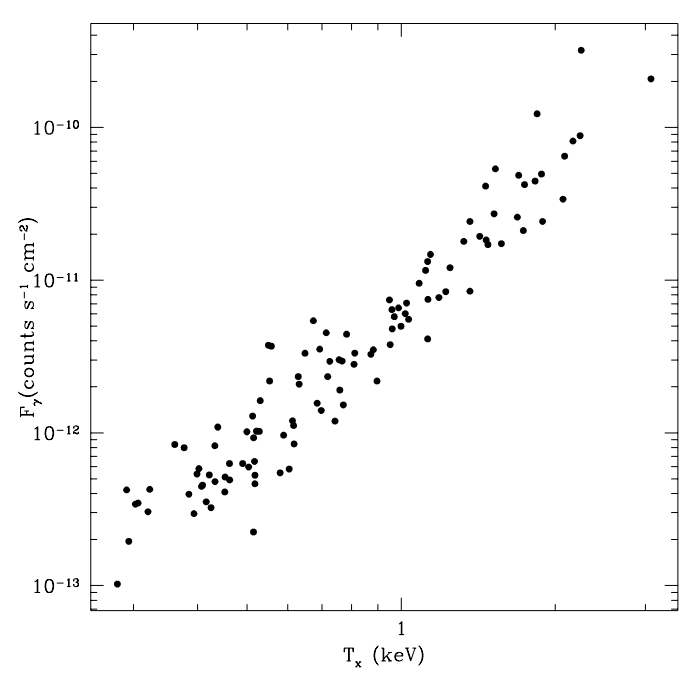

FIGURE 6. High energy $\gamma$-ray flux $(>100 \mathrm{MeV})$ from simulated clusters at $z=0$ assuming a distance $70 \mathrm{Mpc} / \mathrm{h}$, with $\mathrm{h}=0.5$. 
shocks, while the thermal density increases smoothly towards cluster centers. The rate of $\gamma$-ray production from pion decay depends on the collision rate between thermal and CR protons, so scales as the sum of these two (logarithmic) images. Thus, the $\gamma$ ray emission may have extensions beyond the thermal X-rays, and may show fine structure not evident in the thermal emission, as well. As mentioned earlier, most of the shock processing of the gas in clusters involves shocks with Mach numbers $\sim 5$ or so. Thus, the computed CR energy distributions typically resemble $N(E) \propto E^{-2.1}$, and the predicted $\gamma$-ray spectrum has a similar form.

In Figure 6 we show the simulated $\gamma$-ray flux, $F_{\gamma}(>100 \mathrm{MeV})$, emitted within 1.3 $\mathrm{Mpc} / \mathrm{h}$ of cluster centers as a function of the cluster temperature, $T_{x}$, assuming each is at the distance of the Coma cluster, $70 \mathrm{Mpc} / \mathrm{h}$ with $\mathrm{h}=0.5$. The least squares fit to this distribution gives $F_{\gamma} \propto T_{x}^{2.95}$. A scaling close to $F_{\gamma} \propto T_{x}^{3}$ makes sense in this model from the fact that the kinetic power in accretion shocks of virialized clusters scales as $T_{x}^{2}$, providing a scaling for $P_{C R}$ or $n_{C R}$, while the mean cluster baryon densities scale approximately as $T_{x}$ in the simulation. The $F_{\gamma}: T_{x}$ relation found in this calculation is considerably steeper than in some other models, such as that by Colafrancesco \& Blasi [10], which assumes all the CR protons diffuse from a central injection point and predicts closer to $F_{\gamma} \propto T_{x}^{0.5}$. Our simulation utilized a box too small to produce clusters as massive as Coma, but an extrapolation of the $\gamma$-ray relation in Figure 6 to $T_{x}=8.3 \mathrm{keV}$ leads to a prediction of about $3 \times 10^{-9}$ counts $/ \mathrm{sec} / \mathrm{cm}^{2}$, roughly the same as the value found by Colafrancesco and Blasi for the same cluster mass, despite the other differences in the models. The current EGRET limit for Coma is $4 \times 10^{-8}$ [51], so an order of magnitude higher. Still, the coming generation of $\gamma$-ray telescopes should be able to test such models as this. Indeed, those telescopes promise a genuine opportunity to probe the physics of galaxy clusters in telling ways not possible before.

The work of TWJ and FM has been supported in part by NASA grant NAG55055, by NSF grants AST96-16964 and AST00-71167, and by the University of Minnesota Supercomputing Institute. FM also wishes to acknowledge a Doctoral Dissertation Fellowship from the University of Minnesota. DR and HK were supported in part by grant 1999-2-113-001-5 from the interdisciplinary Research Program of the KOSEF.

\section{REFERENCES}

1. Bahcall, N. A., in Formation of Structure in the Universe, eds: A. Dekel \& J. Ostriker, Cambridge: Cambridge University Press, 1999, ch 4, pp. 135-171.

2. Begelman, M. C., Blandford, R. D. \& Rees, M. J., Rev. Mod. Phys. 56, 255 (1984).

3. Berezhko, E. G. \& Ellison, D. C., Astrophys. J. 526, 385 (2000).

4. Berezinsky, V. S., Blasi, P. \& Ptuskin, V. S., Astrophys. J. 487, 529 (1997).

5. Bertschinger, E., Astrophys. J. 58, 39 (1985).

6. Blasi, P., Astrophys. J. 525, 603 (1999). 
7. Böhringer, H., in Extragalactic Radio Sources: Proceedings of the 175th IAU Symposium, ed: R. D./ Ekers \& L. Padrielli, Kluwer, 1996, p 357.

8. Brunetti, G., Setti, G., Feretti, L. \& Giovanini, G., M.N.R.A.S. (in press 2000).

9. Clarke, T. E., Kronberg, P. P. \& Böhringer, H., in Diffuse Thermal and Relativistic Plasma in Galaxy Clusters, ed: H. Böhringer, L. Feretti \& P. Schuecker, Garching: MPE, 1999, p 82.

10. Colafrancesco, S. \& Blasi, P., Astropart. Phys. 9, 227 (1998).

11. Dennison, B., Astrophys. J. 239, L93 (1980).

12. Dolag, K. \& Ensslin, T. A., Astron. E Astrophys. (in press 2000).

13. Donnelly, R. H., Markevitch, M., Forman, W., Jones, C., David, L. P., Churazov, E. \& Gilfanov, M., Astrophys. J. 500, 138 (1998).

14. Drury, L. O'C., Markiewicz, W. J. \& Völk, H. J., Astron. Astrophys. 225, 179 (1989).

15. Dupke, R. A. \& Bregman, J. N., astro-ph/0009429 (2000).

16. Eilek, J. \& Weatherall, J. C., in Diffuse Thermal and Relativistic Plasma in Galaxy Clusters, ed: H. Böhringer, L. Feretti \& P. Schuecker, Garching: MPE, 1999, p 249.

17. Fields, B. D., Olive, K. A., Cassé M. \& Vangioni-Flam, astro-ph/0010121 (2000).

18. Geller, M. J., Diaferio, A. \& Kurtz, M. J., Astrophys. J. 517, L23 (1999).

19. Ensslin, T. A., Biermann, P. L., Kronberg, P. P. \& Wu, X.-P., Astrophys. J. 477, 560 (1997).

20. Ensslin, T. A., in Diffuse Thermal and Relativistic Plasma in Galaxy Clusters, ed: H. Böhringer, L. Feretti \& P. Schuecker, Garching: MPE, 1999, p 275.

21. Harris, D. E., Kapahi, V. K. \& Ekers, R. D., Astron. 8 Ap. Suppl. 39, 215 )1980).

22. Heckman, T. M., in Gas and Galaxy Evolution, eds: J. Hibbard, M. Rupen \& J. van Gorkom, San Francisco: ASP (in press 2000).

23. Jaffe, W. J., Astrophys. J. 212, 1 (1977).

24. Foltz, C. B., Weymann, R. J., Peterson, B. M., Sun, L., Malkan, M. A., \& Chaffee, F. H. Jr., Astrophys. J. 307, 504 (1986)

25. Jones, T. W., Ryu, D. \& Engel, A., Astrophys. J. 512, 105 91999).

26. Jokipii, J. R. \& Morfill, G., Astrophys. J. 312, 170 (1987).

27. Kaastra, J. S., Lieu, R., Mittaz, J., Bleeker, J., Mewe, R., Colafrancesco, S. \& Lockman, F. J., Astrophys. J. 519, 119 (1999).

28. Kang, H. \& Jones, T. W., Astrophys. J. 447, 944 (1995).

29. Kang, H., Ryu, D. \& Jones, T. W., Astrophys. J. 456, 422 (1996).

30. Kang, H., Rachen, J., \& Biermann, P. L., M.N.R.A.S. 286257 (1997).

31. Kang, H., Jones, T. W., LeVeque, R. \& Shyue, K.-M., Astrophys. J. (submitted) (2000).

32. Kikuchi, K., Itoh, C., Kushino, A., Furusho, T., Matsushita, K., Yamasaki, N. Y., Ohashi, T., Fukazawa, Y., Ikebe, Y., Böhringer, H., \& Matsumoto, H., Astrophys. J. 531, L95 (2000).

33. Knopp, G. P., Henry, J. P. \& Boriel, U. G., Astrophys. J. 472, 125 (1996).

34. Kronberg, P. P., Lesch, H., \& Hopp, U., Astrophys. J. 511, 56 (1999).

35. Kronberg, P. P. These proceedings.

36. Kulsrud, R., Cen, R., Ostriker, J. P., \& Ryu, D., Astrophys. J. 480, 481 (1994).

37. Liang, H., in Diffuse Thermal and Relativistic Plasma in Galaxy Clusters, ed: H. Böhringer, L. Feretti \& P. Schuecker, Garching: MPE, 1999, p 33. 
38. Lieu, R., Ip, W.-H., Axford, W. I. \& Bonamente, M. Astrophys. J. 517, 91 (1999)

39. Loewenstein, M., Astrophys. J. 532, 17 (2000).

40. Markevitch, M., Sarazin, C. L. \& Vikhlinin, A., Astrophys. J. 503, 77 (1999).

41. Miniati, F., Ryu, D., Kang, H., Jones, T. W., Cen, R. \& Ostriker, J. P., Astrophys. $J .$, in press (2000).

42. Miniati, F., Ph.D. Thesis, University of Minnesota (2000).

43. Mushotzky, R., in The Hy-Redshift Universe: Galaxy Formation and Evolution at High Redshift, ASP Conference Proceedings, Vol 193, eds: A. J. Bunker \& W. J. van Breugel, San Francisco: ASP, 1999, p 323.

44. Röttgering, H. J. A., Wieringa, M . H., Hunstead, R. W. \& Ekers, R. D., M.N.R.A.S. 290, 577 (1997).

45. Roettiger, K., Burns, J. O., \& Stone, J. M., Astrophys. J. 518, 603 (1999).

46. Ryu, D., \& Kang, H., M.N.R.A.S. 284, 416 (1997).

47. Ryu, D., \& Kang, H. in Proc. of the 18th Texas Symposium on Relativistic Astrophysics, eds: A. Olinto, J. Friedman, \& D. Schramm, 1997, p. 572.

48. Ryu, D., Ostriker, J. P., Kang, H. \& Cen, R., Astrophys. J. 414, 1 (1993).

49. Sarazin, C. L. \& Lieu, R., Astrophys. J. 494, 177 (1998).

50. Sarazin, C. L., Astrophys. J. 520, 529 (1999).

51. Sreekumar, et al., Astrophys. J. 464, 628 (1996).

52. Tribble, P., M.N.R.A.S. 263, 31 (1993).

53. Völk, H. J., Aharonian, F. A., \& Breitschwerdt, D., Space Science Reviews 75, 279 (1996).

54. Völk, H. J. \& Atoyan, A. M., Astroparticle Phys. 11, 73 (1999).

55. Watanabe, M., Yamashita, K., Furuzawa, A., Kunieda, H., Tawara, Y. \& Honda, H., Astrophys. J. 527, 80 (1999).

56. Weymann, R. J., Williams, R. E., Peterson, B. M., \& Turnshek, D. A., Astrophys. J. 234, 33 (1979). 